\title{
Role of platelet rich plasma in chronic plantar fasciitis: An outcome analysis
}

\author{
Snehashish Mukherjee ${ }^{1 *}$, D. K. Taneja ${ }^{2}$, H Safderi $^{3}$ \\ ${ }^{1}$ Junior Resident $3,{ }^{2} \mathrm{HOD},{ }^{3}$ Associate Professor, Dept. of Orthopaedics, Arihant Hospital and Research Centre, Indore, Madhya Pradesh, \\ India
}

*Corresponding Author: Snehashish Mukherjee

Email: dsnehashish@gmail.com

\begin{abstract}
Introduction: plantar fasciitis is most common cause of chronic heel pain. Platelet rich plasma is a new treatment modality that can be beneficial in refractory cases. The purpose of this study is to determine the effectiveness and safety of local PRP injection in plantar fasciitis.

Materials and Methods: Thirty-two cases (23 females and 9 males) were treated by local injection of PRP at heel in Arihant Hospital and Research Centre, Indore between October 2016 to September 2017 and followed-up in this prospective study. AOFAS hind foot score and Roles-Maudsley score were used to determine improvement in pain.

Results: The mean age of the patients was 45.71 \pm 12.1 years (range 23-67 years) and female dominant. Mean duration of symptoms was $11.7 \pm 6.7$ months (range 6 months to 24 months). Only patients having symptoms for more than 3 months with no relief from conservative treatment were involved in study. The initial mean AOFAS hind foot score, $44.41 \pm 3.25$ improved to $91.31 \pm 7.37$ at 9 month follow up, which was statistically significant $(\mathrm{p}<0.05)$. The Roles-Maudsley score improved from poor in all the patients to $90.6 \%$ patients having good to excellent roles-maudsley score at 9 month follow up. Four patients required a repeat injection at 3 months, from which one patient showed no improvement while rest had significant improvement.
\end{abstract}

Conclusion: local injection of Platelet rich plasma is an effective and safe method in refractory cases of plantar fasciitis.

Keywords: Plantar fasciitis, Heel pain, Platelet rich plasma.

\section{Introduction}

Chronic heel pain is one of the most common problems that every clinician faces daily in his day to day Out patient and the exact cause of this is still unknown. Painful heel was first described by Steill in 1922 to be a condition that is seldom efficiently treated because exact cause is not yet known. ${ }^{1}$ After 40 years Lapidus and Guidotti also stated that painful heel is a deliberate term used because cause is still unknown. ${ }^{2}$ Plantar fasciitis is one of the most important causes of heel pain. Woolnough reported it to be affecting 10 percent of general population worldwide. ${ }^{3}$ Etiology of plantar fasciitis is thought to be multifactorial. It is a selflimiting disease. Most commonly used treatment options are shoe inserts with soft sole, anti-inflammatory agents likeNSAIDS and icing locally and plantar fascia stretching exercises. Murphy reported about 90 percent of the patients to get benefitted with these conservative treatments. ${ }^{4}$ But in some cases pain persists despite continuing all these conservative treatments for a long time. Currently widely used treatment options for chronic plantar fasciitis are -local injection of steroid or Platelet rich plasma (PRP) and extracorporeal shock wave therapy (ESWT). Out of all these Platelet rich plasma (PRP) is an emerging concept which focus on healing of injured soft tissue with the help of growth factors present with no adverse effect.

\section{Materials and Methods}

32 patients of chronic planter fasciitis were included in the study presented to us at Arihant hospital and research Centre Indore with in duration from October 2016 to September 2017. This was a prospective cohort study. Patients with Clinical diagnosis of plantar fasciitis with symptoms for more than 3 months with no response to conservative treatments such as non-steroidal anti-inflammatory drugs (NSAIDs) and physiotherapy, shoe changes were included. Any patient with neural entrapment or previous surgery for plantar fasciitis, History of stroke within the last 3 months, and evidence of malignancy, peripheral neuropathy, acute infection, and coagulopathies., Patients with surgical intervention on ankle/heel or any existing foot and ankle pathology - Subtalar arthritis, deformity of ankle and foot, pes planus were excluded. Any patient who had local steroid injection at heel in past 1 year or consumed aspirin or NSAIDs, steroids in the previous week were also excluded.

\section{Method of Preparation}

NSAIDS and oral steroids were stopped in every patient 1 week prior to the injection. Xylocaine sensitivity test was performed in every patient. We have used standard digital centrifuge with maximum range up to $4000 \mathrm{rpm}$ with 8 tubes for centrifugation. Disposable syringes were used for blood collection, and platelet rich plasma injection. For PRP preparation pre-filled anticoagulant mixed tubes were used for centrifugation. We used $3.2 \%$ sodium citrate as an anticoagulant. Following all sterile measures $20 \mathrm{cc}$ venous blood was collected by us from the cubital vein (most commonly) using syringe and transferred immediately to the citrate containing tubes. Tubes were shaken well to mix anticoagulant with the blood to prevent clotting. A double centrifugation system was used - first hard spin done at $1600 \mathrm{rpm}$ for 12 minutes and second $3500 \mathrm{rpm}$ for 12 minutes. In first spin erythrocytes are separated in the basal layer as dark red sediment and white blood cells are separated as upper layer as yellow supernatant. Supernatant was removed carefully and collected in another empty tube. After second spin a whitish yellow platelet rich buffy coat 
layer was obtained at base. The buffy coat layer mainly contains the platelets and white blood cells. Total $3 \mathrm{ml}$ of buffy coat layer with plasma was extracted carefully taking care not to take any erythrocytes. No anticoagulant was used in tubes before second spin. No activator was used in our study.

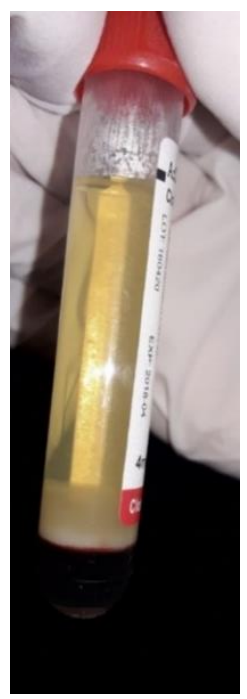

Fig. 1

Fig. 2
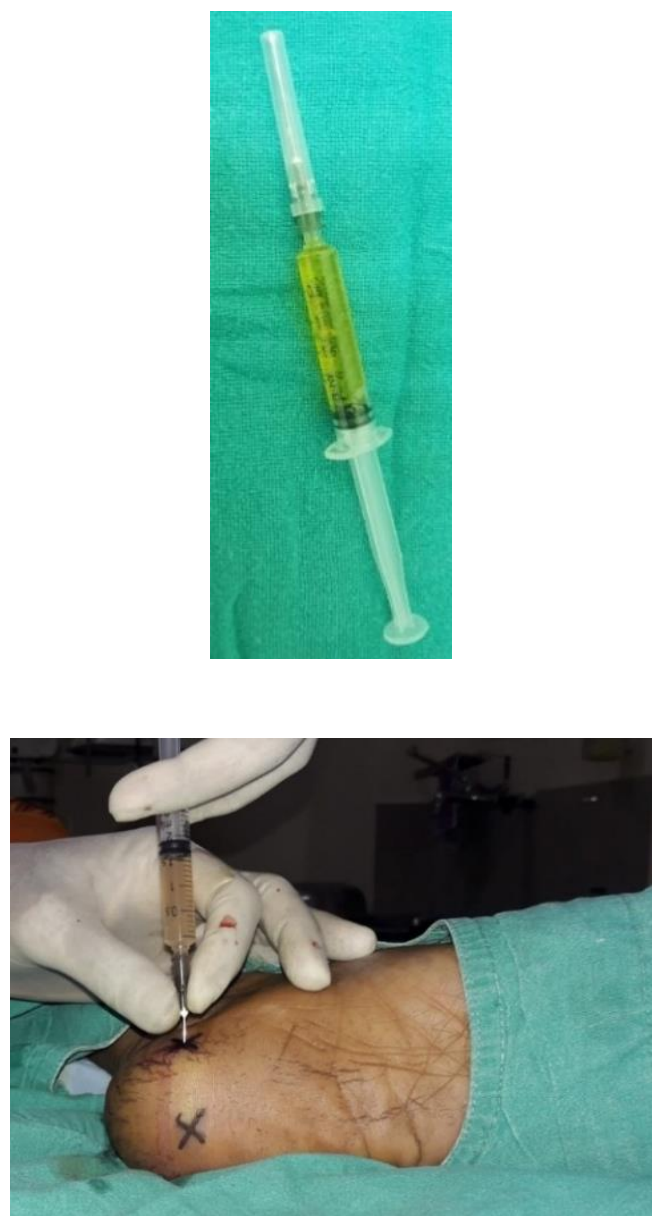

\section{Method of Injection}

All patients were injected with freshly prepared $3 \mathrm{ml}$ of platelet rich plasma injection at heel using a medial entry point with direction towards the most tender point. All patients were injected first with $0.5 \mathrm{ml}$ local anesthetic $(2 \%$ plain xylocaine) before PRP injection. In bilateral cases only one foot is injected at a time and another foot injected after 3 weeks.

\section{Post Injection Protocol}

Oral tramadol/acetaminophen composition for pain relief for 3 days. Any non-steroidal anti-inflammatory drugs (NSAIDs), steroid and icing was avoided for next 7 days, as it hampers the inflammatory mechanism. Briefing done about possibility of increased pain after injection.

\section{Rehabilitation Protocol}

Phase I (0-3 days): Strict non-weight bearing. Only gentle active range of movement exercise (dorsiflexion and plantar flexion) allowed

Phase II (3 days- 6 weeks): Start gradual weight bearing progressing to full weight bearing. Bare foot walking was strictly avoided. Active range of movements were continued plantar fascia stretching exercise started gradually.

Strengthening exercises started at 14 day. Toe walking and wall exercises were started after 14 days about 3-4 sets daily for 5 minutes each time. Slant board exercises also started.

Phase III (after 6 weeks): Strengthening exercises were continued with increased frequency and against resistance. Sports specific exercises were started.

Assessment: Pain improvement was measured by RolesMaudsley (RM) score and functional improvement of foot measured by using American Orthopedic Foot and Ankle Society (AOFAS) hind foot score at 3 weeks, 3 months, 6 months and 9 months.

\section{Results}

From October 2016 to September 2017, 32 patients were included in the study and followed up for 9 months. Only those patients were considered for injection who have symptoms for more than 3 months and got no relief with conservative treatment. Patients who had received steroid injection in past 1 year were also excluded from the study. Study included 23 females $(71.9 \%)$ and 9 males $(28.1 \%)$. Mean age of our patients was 45.71 \pm 12.1 years (range 23-67 years). Most of the patients have left side (59.4\%) involvement. Mean duration of symptoms was $11.7 \pm 6.7$ months (range 6 months to 24 months).

All the patients had "poor" Pre injection roles-maudsley score, which improved significantly to $90.6 \%$ patients having good to excellent roles-maudsley score at 9 month post injection. Final results is shown below:

Fig. 3 
Table 1

\begin{tabular}{|l|c|c|c|c|c|}
\hline Roles Maudsley Score & $\begin{array}{c}\text { No of patients at } \\
\text { presentation }\end{array}$ & $\begin{array}{c}\text { No of patients } \\
\text { at 3 weeks }\end{array}$ & $\begin{array}{c}\text { No of patients } \\
\text { at 3 months }\end{array}$ & $\begin{array}{c}\text { No of patients } \\
\text { at 6 months }\end{array}$ & $\begin{array}{c}\text { No of patients } \\
\text { at 9 months }\end{array}$ \\
\hline Poor & 32 & 8 & 2 & 1 & 1 \\
\hline Acceptable & 0 & 10 & 4 & 2 & 2 \\
\hline Good & 0 & 14 & 16 & 19 & 19 \\
\hline Excellent & 0 & 0 & 10 & 10 & 10 \\
\hline Total & 32 & 32 & 32 & 32 & 32 \\
\hline
\end{tabular}

Pre injection mean AOFAS hind foot score was $44.41 \pm 3.25$ in our study, which improved gradually to $78.94 \pm 8.23$ at 3 weeks, $88.25 \pm 9.11$ at 3 months, $90.78 \pm 7.69$ at 6 months and $91.31 \pm 7.37$ at 9 months which was significant $(p$ value= $0.003)$.

Table 2: Association of duration of symptoms with Roles Maudsley score at 9 months

\begin{tabular}{|c|c|c|c|c|c|}
\hline Duration of symptoms & \multicolumn{4}{|c|}{ Roles Maudsley Score at 9 months } & Total \\
\hline & Poor & Acceptable & Good & Excellent & \\
\hline 6-12 months & 0 & 1 & 14 & 8 & 23 \\
\hline $13-18$ months & 0 & 1 & 0 & 2 & 3 \\
\hline 19-24 months & 1 & 0 & 5 & 0 & 6 \\
\hline Total & 1 & 2 & 19 & 10 & 32 \\
\hline
\end{tabular}

Pearson chi-square value $=13.903, d f=6, p$ value $=0.031$, Significant

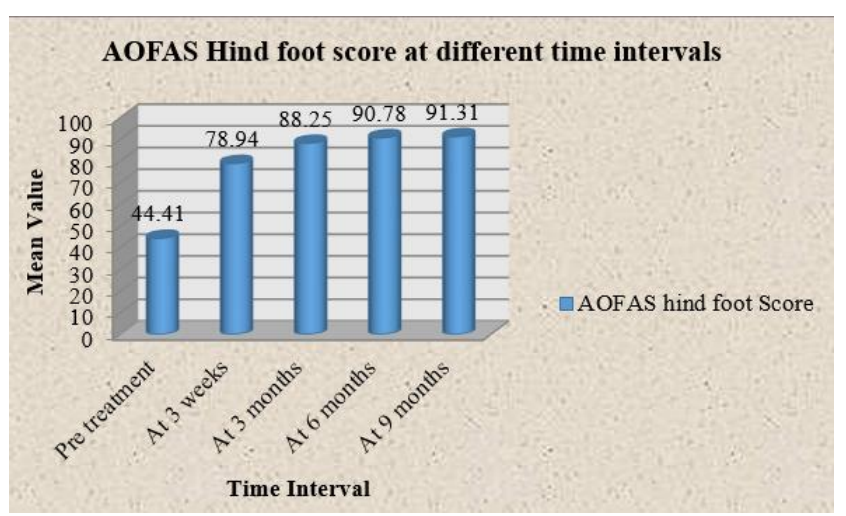

Fig. 4

At 3 months after the first injection 4 patients (12.5\%) had no improvement in pain so they were given a repeat local PRP injection at same site. At 9 month follow up in these patients 1 patient still had "poor" Roles Maudsley score, 2 had "acceptable" score and 1 had "good" score.

Out of 32 patients, only $5(15.6 \%)$ patients had injection site pain for initial 3-4 days which relieved gradually and it does not affect the final result. For pain relief in these cases acetaminophen was used. No infection or any other complication noted in our study. Pre-injection 20 patients $(62.5 \%)$ had walking difficulty at morning due to severe pain which reduced to only 1 patient $(3.1 \%)$. Final roles-maudsley score at 9 months had a statistically significant association with duration of symptoms of the patients $(\mathrm{p}$ value $=0.031$ )

\section{Discussion}

Plantar fasciitis has a significant negative impact on social health and causes increased economic burden on society. Most commonly accepted view in literature for etiopathology is that it is a result of repeated micro tears and cystic degeneration in plantar fascia. Lemont et al (2003) $)^{5}$ has proposed the term "plantar fasciosis" for the entity on histological findings of degenerative changes.

Most common modes of treatment are conservative in form of shoe inserts, anti-inflammatory drugs and icing, plantar fascia stretching exercises which provide good results in about 90 percent of the patients. ${ }^{4}$ Local steroid injection are used for pain relief in chronic cases but Crawford et $\mathrm{al}^{6}$ had shown that steroid injections provide short term relief. Steroid also has some side effects like: infection, increased blood sugar, fat pad atrophy ${ }^{7}$ and planter fascia rupture. ${ }^{8}$

Platelet rich plasma is a good alternative of steroid in these patients. In clinical practice, Ferrari et $\mathrm{al}^{9}$ in 1987 first used PRP autologous transfusion component in open heart surgery. First musculoskeletal use of PRP was reported by Sanchez et al ${ }^{10}$ at 2003 when they published a case report of using autologous growth factor rich plasma in articular cartilage avulsion.

PRP contains approximately three to five times increased concentration of growth factors. Harrison et $\mathrm{al}^{11}$ have demonstrated that in vivo collagen activation by PRP leads to sustained release of growth factors over 7 days. The properties of PRP are due to the production and release of multiple growth and differentiation factors from $\alpha$-granules when platelets are activated. Growth factors promote angiogenesis and fibroblast proliferation, needed for tendon repair and promotes chemotaxis of macrophages and fibroblasts and increases cellular activity in early healing stages. It also enhances fibronectin and glycosaminoglycan deposition and collagen synthesis. Anitua et al (2009) ${ }^{12}$ demonstrated in their study that fibroblast proliferation in skin, synovium and tendon, is simulated by growth factor rich formulations and it depends on the concentration of platelets. 
Proper post injection protocol and rehabilitation protocol plays an essential part in getting good results. NSAIDs have an anti-inflammatory effect, so they were avoided in immediate post-injection period and prior to injection also. We have kept patient on non-weight bearing for 3 days and avoided any exercises during this period to give the limb rest. Any splint or boot cast was not used in our study to immobilize the patient for first three days. Aggressive running, jumping was avoided for 2 weeks.

Local PRP injection sets up a new inflammatory process which may cause increased pain for 3-4 days but subsides eventually and does not affect final result. It takes about 3 weeks for the healing to occur and pain subsides, but in some cases it may take even more time up to 6 weeks. All patients were advised to continue wearing shoe inserts or soft shoes after the injection. Plantar fascia stretching exercises started gradually 3 days after injection and advised to continue exercise in follow up period.

In our study pre-procedure AOFAS hind foot score was $44.41 \pm 3.25$ which improved to $91.31 \pm 7.37$ at 9 months . Monto et al (2014) $)^{13}$ performed a comparative study between local platelet rich plasma (PRP) injection and local steroid injection in cases of chronic plantar fasciitis including 36 patients. Theirs pre injection AOFAS hind foot score of 37 improved to 95 at 3 months and 94 at 6 and 12 month after injection. They also had no complication in their study. In comparable to study of Mahindra et al $(2016)^{14}$ where pre-procedure AOFAS hind foot score improved from pre-procedure score of 51.56 to 88.24 at 3 month.

At 9 months Roles- Maudsley score showed significant improvement with 29 cases having "good to excellent" results. Martinelli et al (2013) ${ }^{15}$ in their study of 14 people observed results at 12 month follow up as $9(64.3 \%)$ patients as excellent, $2(14.3 \%)$ as good and $2(7.1 \%)$ as acceptable and 1 patient $(7.1 \%)$ as poor on Roles Maudsley score. They also noticed post procedural pain as a complication in their study.

These 3 failed cases were further reevaluated and thoroughly assessed clinically and radio graphically. Their pre-injection and post injection status compared but we did not find any specific cause for failure, but it may be due to non-compliance of the patients to the rehabilitation regime. These patients were advised that surgery may be an option in future. In our study we also noticed that duration of symptoms had a significant relationship with the final outcome $(p=0.031)$. In long term cases many chronic changes occur at plantar fascia which may be the cause of lesser results in these cases. So early injections have better results.

Limitations of our study was that all the patients were diagnosed and improvement assessed by clinically and on subjective scores (AOFAS hind foot score and Roles and maudsley score). More significant results can be observed in a larger population with longer duration of follow up, which we could not perform in our study due to time constraint.

\section{Conclusion}

Thus local injection of PRP can be considered as good and effective alternative to steroid for chronic plantar fasciitis with no major complications. Cases for local PRP injections should be chosen wisely and rule out all other possibilities of chronic heel pain to obtain good results. It's better to prepare PRP just before the procedure and try to inject it as early as possible to have maximum growth factor concentration. Post injection exercise and shoe inserts formed a vital part of rehabilitation in our patients.

\section{Conflict of Interest: None.}

\section{References}

1. Stiell WF. Painful heel. Practitioner 1922;108:345.

2. Lapidus PW, Guidotti FP. Painful heel: report of 323 patients with 364 painful heels. Clin Orthop 1965;39:178-86.

3. Woolnough J. Tennis heel. Med J Aus 1954;2:857-61.

4. Murphy GA. Disorders of tendons and fascia and adolescent and adult pes planus. Chapter 82. In: Campbell's operative orthopaedics. 12th ed. Philadelphia: Elsevier Mosby; 2013;1:3954-5.

5. Lemont H, Ammirati KM, Usen N. Plantar fasciitis: a degenerative process (fasciosis) without inflammation. $J$ Am Podiatr Med Assoc 2003;93:234-7.

6. Crawford F, Thomson C. Interventions for treating plantar heel pain (Review). Cochrane Database Syst Rev 2003;(3):CD000416.

7. Acevedo JI, Beskin JL. Complications of plantar fascia rupture associated with corticosteroid injection. Foot Ankle Int 1998;19:91-7.

8. Buccilli TA Jr, Hall HR, Solmen JD. Sterile abscess formation following a corticosteroid injection for the treatment of plantar fasciitis. J Foot Ankle Surg 2005;44:466-8.

9. Ferrari M, Zia S, Valbonesi M, Henriquet F, Venere G, Spagnolo S, et al. A new technique for hemodilution, preparation of autologous platelet-rich plasma and intraoperative blood salvage in cardiac surgery. Int J Artif Organs 1987;10(1):47-50.

10. Sánchez M, Azofra J, Anitua E, Andía I, Padilla S, Santisteban $\mathrm{J}$, et al. Plasma rich in growth factors to treat an articular cartilage avulsion: a case report. Med Sci Sports Exerc 2003;35(10):1648-52.

11. Harrison S, Vavken P, Kevy S, Jacobson M, Zurakowski D, Murray MM. Platelet activation by collagen provides sustained release of anabolic cytokines. Am J Sports Med 2011;39(4):729-34.

12. Anitua E, Sanchez M, Zalduendo MM, de la Fuente M, Prado R, Orive G, et al. Fibroblastic response to treatment with different preparations rich in growth factors. Cell Prolif 2009;42(2):162-70.

13. Monto RR. Platelet-Rich Plasma Efficacy versus Corticosteroid Injection Treatment for Chronic Severe Plantar Fasciitis. Foot Ankle Int 2014;35(4):313-8.

14. Mahindra P, Yamin M, Selhi H, Singla S, Soni A. Chronic Plantar Fasciitis: Effect of Platelet-Rich Plasma, Corticosteroid, and Placebo. Orthopedics. 2016;39:e285-e289.

15. Martinelli N, Marinozzi A, Carnì S, Trovato U, Bianchi A, Denaro V. Platelet-rich plasma injections for chronic plantar fasciitis. Int Orthop 2013;37(5):839-42.

How to cite this article: Mukherjee S, D. K. Taneja, H Safderi. Role of platelet rich plasma in chronic plantar fasciitis: An outcome analysis. Indian J Orthop Surg 2019;5(2):114-7. 